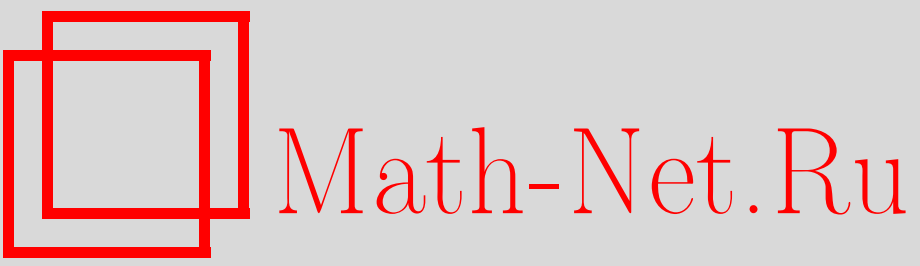

М. Е. Чанга, Суммирование мультипликативных функций, УМH, 2002, том 57, выпуск 6, 197-198

DOI: https://doi.org/10.4213/rm590

Использование Общероссийского математического портала Math-Net.Ru подразумевает, что вы прочитали и согласны с пользовательским соглашением

http://www.mathnet.ru/rus/agreement

Параметры загрузки:

IP : 52.23 .180 .231

26 апреля 2023 г., 14:43:16 


\title{
СУММИРОВАНИЕ МУЛЬТИПЛИКАТИВНЫХ ФУНКЦИЙ
}

\author{
М. Е. ЧАНГА
}

Напомним вначале некоторые определения теории мультипликативных функций $[1 ;$ с. 316$]$.

ОпредЕлЕниЕ. Мултипликативная функция $f(n)$ назьвается вполне мультипликативной, если соотношение $f(a b)=f(a) f(b)$ верно для любых натуралшных $a$ и $b$.

ОпредЕлЕниЕ. Мултипликативная функция $f(n)$ назьвается сильно мультипликативной, если для любого простого $p$ и для любого натурального $\alpha$ верно соотношение $f\left(p^{\alpha}\right)=f(p)$.

Основным резултатом данной работы является следующая

Tеорема. Пусть $f(n)=f_{1}(n) f_{2}(n)$, дде $f_{1}(n)$ вполне мультипликативна, а $f_{2}(n)$ сильно мультипликативна. Пусть $F(x)$ обозначает сумматорную функцию $f(n)$. Пусть задано челое число $k$, большее единицы, с каноническим разложением $p_{1}^{\alpha_{1}} \cdots p_{r}^{\alpha_{r}}$. Тогда имеет место равенство

$$
\sum_{n \leqslant x} f(k n)=f(k) \sum_{l_{1}, \ldots, l_{r} \geqslant 0} \omega_{1}^{l_{1}} \cdots \omega_{r}^{l_{r}} F\left(\frac{x}{p_{1}^{l_{1}} \cdots p_{r}^{l_{r}}}\right)
$$

əде $\omega_{i}=f_{1}\left(p_{i}\right)-f\left(p_{i}\right)$ c $i=1, \ldots, r$.

ДокАЗАТЕльство. Из вышеприведенных определений следует, что равенство

$$
f(a b)=\frac{f(a) f(b)}{f_{2}((a, b))}
$$

имеет место для любых натуральных $a$ и $b$. Пусть $p$ - простое число, $\omega=f_{1}(p)-f(p)$ и $m-$ натуральное число, не делящееся на $p$. Тогда справедливо соотношение

$$
\sum_{n \leqslant x} f(p m n)=f(p) \sum_{l \geqslant 0} \omega^{l} \sum_{n \leqslant x / p^{l}} f(m n) .
$$

Действительно, пользуясь формулой (1) и тем, что $(p, n)=1$ при $p \nmid n$ и $(p, n)=p$ при $p \mid n$, имеем

$$
\sum_{n \leqslant x} f(p m n)=f(p) \sum_{\substack{n \leqslant x \\ p \nmid n}} f(m n)+f_{1}(p) \sum_{\substack{n \leqslant x \\ p \mid n}} f(m n)=f(p) \sum_{n \leqslant x} f(m n)+\omega \sum_{n \leqslant x / p} f(p m n) .
$$

Повторяя ту же процедуру с последней суммой в правой части, за конечное число шагов приходим к соотношению (2). Пусть $\alpha$ - натуральное число. Тогда имеет место равенство

$$
\sum_{n \leqslant x} f\left(p^{\alpha} m n\right)=f\left(p^{\alpha}\right) \sum_{l \geqslant 0} \omega^{l} \sum_{n \leqslant x / p^{l}} f(m n) .
$$

Действуя аналогично предыдущему, находим при $\alpha \geqslant 2$

$$
\begin{aligned}
\sum_{n \leqslant x} f\left(p^{\alpha} m n\right) & =f\left(p^{\alpha}\right) \sum_{\substack{n \leqslant x \\
p \nmid n}} f(m n)+f_{1}\left(p^{\alpha}\right) \sum_{\substack { \beta=1 \\
\begin{subarray}{c}{n \leqslant x \\
p^{\beta} \| n{ \beta = 1 \\
\begin{subarray} { c } { n \leqslant x \\
p ^ { \beta } \| n } }\end{subarray}}^{\alpha-1} f(m n)+f_{1}\left(p^{\alpha}\right) \sum_{\substack{n \leqslant x \\
p^{\alpha} \mid n}} f(m n) \\
& =f\left(p^{\alpha}\right) \sum_{\substack{n \leqslant x \\
p \nmid n}} f(m n)+f_{1}\left(p^{\alpha}\right) \sum_{\substack{n \leqslant x \\
p \mid n}} f(m n) \\
& =f\left(p^{\alpha}\right) \sum_{n \leqslant x} f(m n)+\left(f_{1}\left(p^{\alpha}\right)-f\left(p^{\alpha}\right)\right) \sum_{n \leqslant x / p} f(p m n) .
\end{aligned}
$$


Применение формулы (2) к последней сумме в правой части приводит нас к соотношению (3). Положим $m_{1}=p_{2}^{\alpha_{2}} \cdots p_{r}^{\alpha_{r}}$ и восполшзуемся формулой (3):

$$
\sum_{n \leqslant x} f(k n)=\sum_{n \leqslant x} f\left(p_{1}^{\alpha_{1}} m_{1} n\right)=f\left(p_{1}^{\alpha_{1}}\right) \sum_{l_{1} \geqslant 0} \omega_{1}^{l_{1}} \sum_{n \leqslant x / p_{1}^{l_{1}}} f\left(m_{1} n\right) .
$$

Далее, положим $m_{2}=p_{3}^{\alpha_{3}} \cdots p_{r}^{\alpha_{r}}$ и применим соотношение (3) к внутренней сумме в правой части. Повторяя эту процедуру $r$ раз и пользуясь мульипликативностью функции $f(n)$, получим утверждение теоремы. Этим доказательство завершается.

Приведем некоторые примеры использования этой теоремы, связанные с мултипликативной функцией Эйлера $\varphi(n)$. Первое следствие представляет собой частный случай утверждения, приведенного в $[1 ;$ с. 262].

СлеДСтвиЕ 1. При $x \rightarrow \infty$ имеет место асимптотическая формула

$$
\sum_{n \leqslant x} \varphi(k n)=\frac{3 k}{\pi^{2}} \prod_{p \mid k} \frac{p}{p+1} x^{2}+O(x \ln x),
$$

где постоянная в знаке $О$ зависит только от $k$.

ДокАЗАТЕЛЬСтво. Известно (см., например, [2; с. 30]), что

$$
\varphi(n)=n \prod_{p \mid n}\left(1-\frac{1}{p}\right) .
$$

Таким образом, функция Эйлера удовлетворяет условию теоремы с $f_{1}(n)=n$ и $\omega_{i}=1$. Согласно $[1 ;$ с. 259$]$ имеет место асимптотическая формула

$$
F(x)=\sum_{n \leqslant x} \varphi(n)=\frac{3}{\pi^{2}} x^{2}+O(x \ln (1+x)),
$$

причем оценка остаточного члена равномерна при $0 \leqslant x<\infty$. Отсюда получаем требуемое.

СлЕДСтвИЕ 2. При $x \rightarrow \infty$ имеет место асимптотическая формула

$$
\sum_{n \leqslant x} \frac{1}{\varphi(k n)}=\frac{\zeta(2) \zeta(3)}{k \zeta(6)} \prod_{p \mid k} \frac{p^{2}}{p(p-1)+1} \ln x+O(1),
$$

где постоянная в знаке $O$ зависит только от $k$.

ДоКАЗАТЕЛЬСТво. Согласно соотношению (4) функция $1 / \varphi(n)$ удовлетворяет условию теоремы с $f_{1}(n)=1 / n$ и $\omega_{i}=1 /\left(p_{i}\left(1-p_{i}\right)\right)$. Согласно [3; с. 83] имеем

$$
F(x)=\sum_{n \leqslant x} \frac{1}{\varphi(n)}=\frac{\zeta(2) \zeta(3)}{\zeta(6)} \ln x+O(1),
$$

причем оценка остаточного члена равномерна при $1 \leqslant x<\infty$. Если же $x<1$, то $F(x)=0$. Отсюда получаем требуемое.

\section{СПИСОК ЛИТЕРАТУРЫ}

[1] А.Г. Постников. Введение в аналитическую теорию чисел. М.: Наука, 1971. [2] И. М. Виноградов. Основы теории чисел. М.: Наука, 1981. [3] А. А. Карацуба. Основы аналитической теории чисел. М.: Наука, 1983. 